\title{
A 12 Bit IF Sampling Pipelined ADC in 0.18um BiCMOS
}

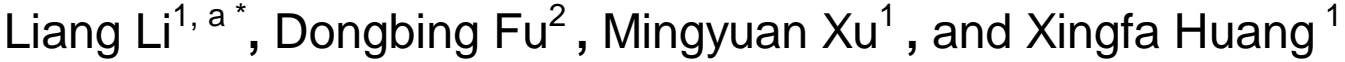 \\ ${ }^{1}$ Science and Technology on Analog Integrated Circuit Laboratory, Chongqing, 400060, China \\ ${ }^{2}$ SISC, CETC, Chongqing 400060, China \\ astanderli@sina.com
}

Keywords: switched capacitor, IF Sampling, input buffer, BiCMOS.

Abstract. In this paper, a 12 bit 500MS/s IF Sampling ADC is described. The ADC has an integrated input buffer with a new linearization technique that improves its distortion. Eight pipeline stages with fully differential switched capacitor architecture follow the input buffer. Each of stage of the pipeline, excluding the last, consists of a low resolution flash ADC connected to a switched capacitor DAC and interstage residue amplifier(MDAC). A $0.18 \mu \mathrm{m}$ BiCMOS process with $1.8 \mathrm{~V}$ analog power supply is used in the design. This ADC achieves an SNR of $65 \mathrm{~dB}$ and an SFDR of $82 \mathrm{~dB}$ for sampling analog input frequencies up to $250 \mathrm{MHz}$

\section{Introduction}

Many communication and signal processing system are demanding $>60 \mathrm{~dB}$ dynamic range while sampling intermediate frequencies that may range from $100 \mathrm{MHz}$ to $500 \mathrm{MHz}$. This paper describes a 12-bit 500MS/s ADC with high linearity input buffer. Section 2 gives a detailed overview of the architecture and describes all key building blocks. Section 3 gives a detailed circuit description of the input buffer, pipelined ADC stages, residue amplifier. Section 4 gives experimental results of the ADC.

\section{Architecture Overview}

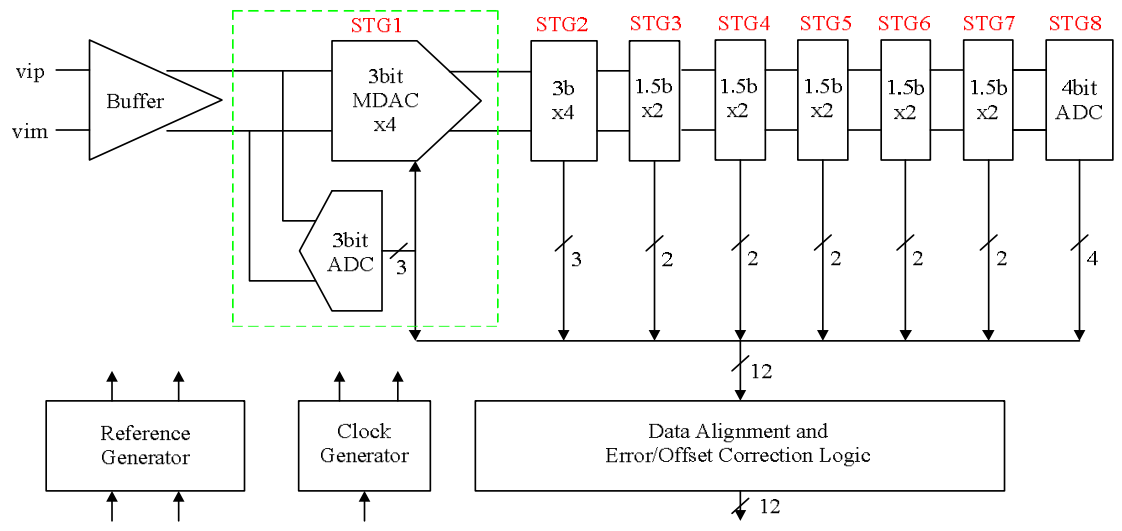

Fig. 1 12-bit ADC architecture

A block diagram of the 12-bit ADC architecture is shown in Fig.1. This SHA-less ADC consists of a differential input buffer followed by eight pipeline stages. The differential input buffer accepts a $1.5 \mathrm{Vp}$-p differential signal. Following the input buffer is a 3-bit MDAC with 1 bit of redundancy to improve comparator offset tolerance. The 3-bit MDAC is followed by another 3-bit MDAC with 1 bit of redundancy and 5 1.5b/stages. The final 4 lsb's are converted by the backend 4 bit flash converter. Each stage of the pipeline consists of sub ADC、DAC and residue amplifier. The residue amplifier magnifies the difference between the reconstructed DAC output and the flash input for the next stage in the pipeline. One bit of redundancy is used in each stage to facilitate digital correction of flash errors. The last stage simply consists of a flash ADC.

This ADC also includes reference and clock generator. A differential voltage reference creates positive and negative reference voltages that define the $1.5 \mathrm{Vp}-\mathrm{p}$ fixed span of the ADC core. A clock 
circuit based on dynamic delay line (DLL) provides all kinds of phases clock to every part of the pipelined ADC.

\section{Key Building Blocks}

\section{Input Buffer Circuit}

Today the high-speed and high-resolution ADC often adopts SHA-less architecture to reduce power, which requires a input buffer with wide bandwidth and high linearity. Also, Adding an input buffer at the ADC front end has the advantage of isolating ADC sampling glitch of the switched capacitor circuit from affecting the external ADC driver circuit. A broadband buffer is relatively easy to implement with emitter follower in a BiCMOS process as high speed NPN devices are available.

High linearity input buffer should have high input impedance and low output impedance, High input impedance can isolate input driver circuit from noise interference produced by sampling switch. Low output impedance can decrease distortion produced by nonlinear load impedance.

This paper proposes a input buffer which can decrease relativity between the $g_{m}$ of input transistor and input siginal, makes the $g_{m}$ of input transistor invariable to input siginal, even keeps a constant. It is well known that the $\mathrm{g}_{\mathrm{m}}$ of input transistor is decided by its bias current, the bias current of input transistor keeps invariable, so that the $\mathrm{g}_{\mathrm{m}}$ of input transistor keeps invariable. Figure 2 is the input buffer with repilca load impedance proposed by this paper. It includes a input transistor NPN Q1, a load capacitor $\mathrm{C}_{\mathrm{L}}$, a replica load capacitor $\mathrm{C}_{\mathrm{RL}}$, a constant current source I constituted by a NPN Q2 and a NMOS M2.

Adopting the input buffer with replica load impedance proposed by this paper, the AC small siginal current $i_{L}$ following input siginal variety on the load capacitor can be provided by siginal source via replica load capacitor. This guarantees that the bias current of input transistor NPN Q1 keeps invariable, so that the $\mathrm{g}_{\mathrm{m}}$ of input transistor NPN Q1 keeps invariable and output siginal distortion will be decreased.

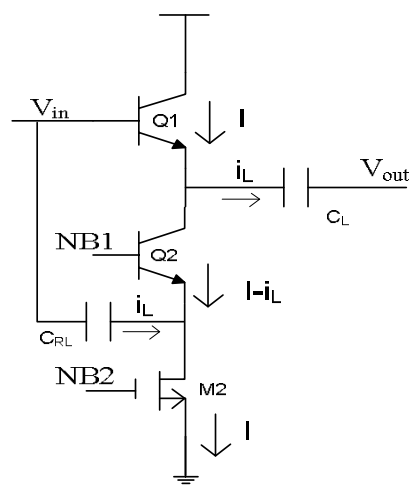

Fig.2 Input buffer with replica load impedance

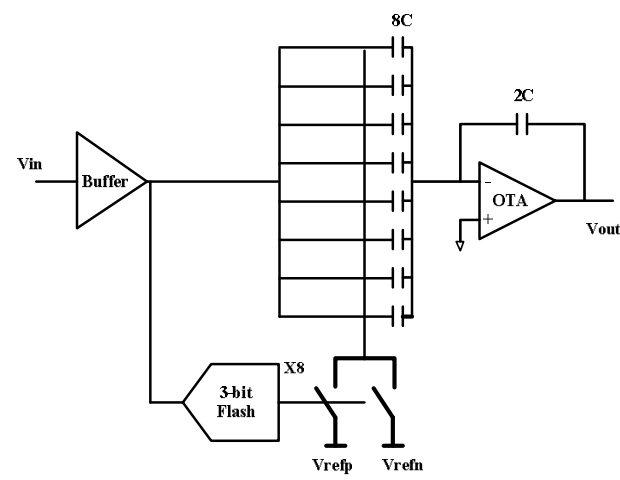

Fig.3 $1^{\text {st }}$ stage pipeline architecture

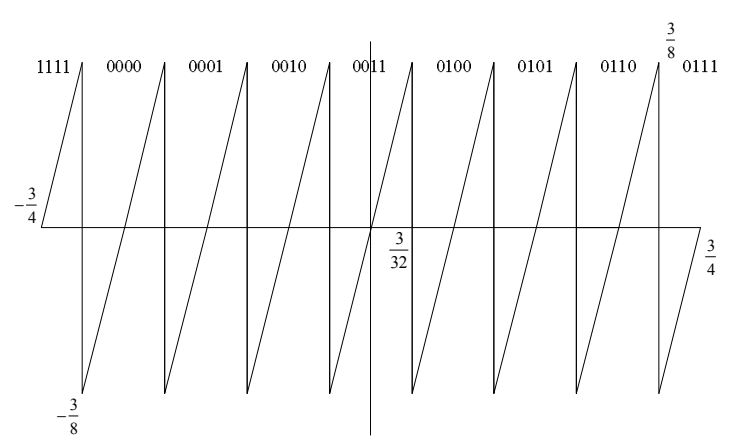

Fig.4 $1^{\text {st }}$ stage output residue curve

\section{Pipelined ADC Stages}

This ADC have a SHA-less analog front end. Followed the low distortion input buffer, a 3 bit architecture is chosen for the ADC's $1^{\text {st }}$ stage. One bit redundancy is added to increase the sub-ADC 
comparator offset tolerance. Multi-bit/stage architecture has been widely used in high speed and high resolution pipelined ADC designs, especially in ADC's front end stages. It has the advantage of reducing the capacitor matching requirement, improve $\mathrm{ADC}$ linearity. Fig. 3 shows the $1^{\text {st }}$ stage pipeline architecture.

The $1^{\text {st }}$ stage flash ADC has sampling networks for its comparators that closely match that of the MDAC. This is needed because any mismatch in the comparators causes the input value sampled by the MDAC to be different from that sampled by the comparators. This leads to flash offset and gain errors that get worse with increasing the input frequency. This error consumes a portion of the correction range of the first stage residue, but can be corrected by the digital error correction only as long as the residue does not exceed the correction range. During the sampling phase, the input is sampled on the MDAC and the flash sampling capacitances. During the hold phase, the flash comparators make their decisions and propagate them to the DAC switches, which connect the DAC capacitances to either Vrefp or Vrefn depending on the flash bits. Fig.4 shows the $1^{\text {st }}$ stage output residue curve.

The ADC's $2^{\text {nd }}$ pipeline stage is also a 3bit stage with 1 bit redundancy. 8 comparators are used in its sub-ADC. According to pipelined ADC theory, the $2^{\text {nd }}$ pipeline stage circuit requirement is relaxed by the gain of the $1^{\text {st }}$ pipeline stage. Although the architecture of the $2^{\text {nd }}$ pipeline stage is same to the $1^{\text {st }}$ pipeline stage, the size of sampling capacitances and switches becomes smaller, the adoption of residue amplifier is different and power consumption decreases.

$1.5 \mathrm{~b} /$ stage architecture is used in stage 3 7. As errors introduced in stage 3 are attenuated by the front stage gain of 16, the noise and matching requirement in these stages are greatly relaxed. Only 8 bit matching accuracy is required for $3^{\text {rd }}$ stage MDAC, even less is needed for later stages. So MDAC capacitors can be scaled down to improve the circuit speed, and reduce ADC power consumption. Fig. 5 shows the 1.5b/stage architecture. Fig. 6 shows its output residue curve.

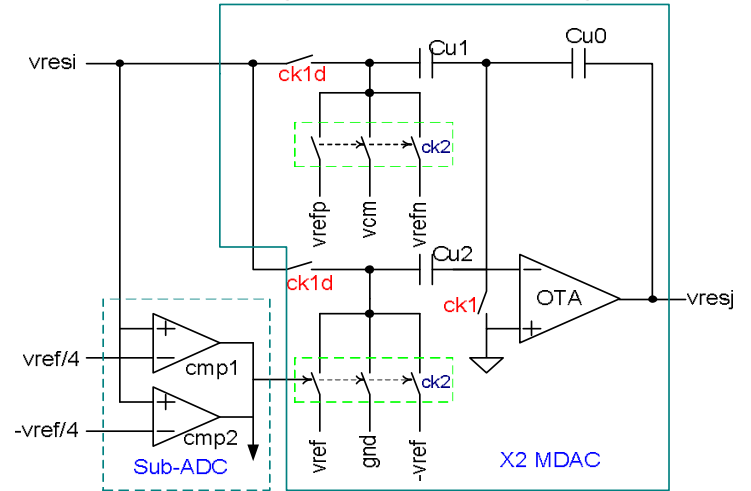

Fig.5 1.5b/stage architecture

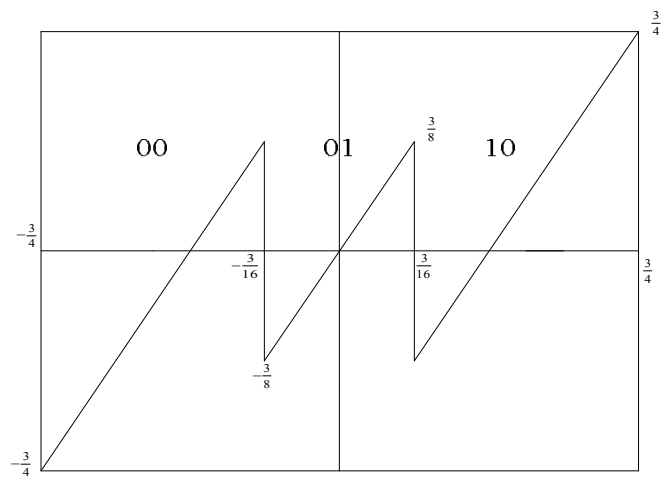

Fig.6 1.5b/stage output residue curve

The last stage is simply a 4bit flash ADC, as no residue voltage needs to be generated for further processing, so the MDAC is removed. 14 comparators are used in the last stage and its outputs are coded from 0000 to 1111 .

\section{Residuse Amplifier Design}

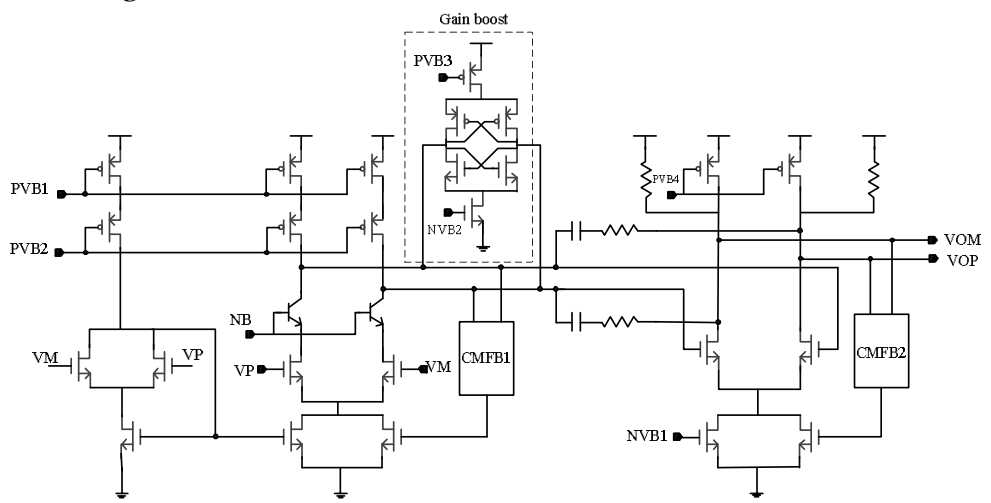

Fig.7 $1^{\text {st }}$ stage pipeline residue amplifier 
The $1^{\text {st }}$ stage pipeline residue amplifier is shown in Fig.7 and consists of two stages. The first stage is a cascoded amplifier with double-cascoded PMOS and BJT-cascoded NMOS. The first stage has also a gain boost block. This circuit uses positive feedback, through cross-coupling, to create a negative trans-conductance circuit in parallel with the main devices. The second stage is a simple differential pair with PMOS current source and resistor load. The Miller compensation capacitances are connected between the outputs of the second stage and the outputs of the first stage. The first stage and the second stage have each a common mode feedback (CMFB) block. The bandwidth required for the $1^{\text {st }}$ stage pipeline residue amplifier to achieve 12-bit performance is about $1.2 \mathrm{GHz}$ and the open loop gain needs to be larger than $80 \mathrm{~dB}$. This required high gain-bandwidth product leads to high power consumption in $1^{\text {st }}$ stage pipeline.

The $2^{\text {nd }}$ stage pipeline residue amplifier architecture is different to the $1^{\text {st }}$ stage pipeline residue amplifier. Because the requirement of $2^{\text {nd }}$ stage pipeline residue amplifier is relaxed, the BJT NPN is no more needed. It is replaced by NMOS. The residue amplifier architecture in stage 3 7 is same to the $2^{\text {nd }}$ stage pipeline residue amplifier. But their sizes and power consumption are scaling down along with pipeline stages according to pipelined ADC theory.

\section{Experimental Results}

The 12 bit ADC is fabricated on a $0.18 \mu \mathrm{m}$ BiCMOS process. Fig. 8 shows an FFT Spectrum of a 30.3MHz sinewave sampled at 500MSPS. SNR is 65.6dB and SFDR is $82.1 \mathrm{~dB}$. Fig. 9 shows DNL and INL of this ADC. The 12bit ADC performance is summaried in the following table.

Table 1 This ADC performance summary

\begin{tabular}{|l|l|}
\hline Technology & $0.18 \mu \mathrm{m}$ BiCMOS \\
\hline Supply Voltage & $1.8[\mathrm{~V}]$ \\
\hline Differential Input Range & $1.5[\mathrm{Vp}-\mathrm{p}]$ \\
\hline DNL/ INL & $\pm 0.4[\mathrm{LSB}] / \pm 0.9[\mathrm{LSB}]$ \\
\hline SNR/SFDR & $65.6[\mathrm{~dB}] / 82.1[\mathrm{~dB}]$ \\
\hline Power Dissipation & $700[\mathrm{~mW}]$ \\
\hline
\end{tabular}

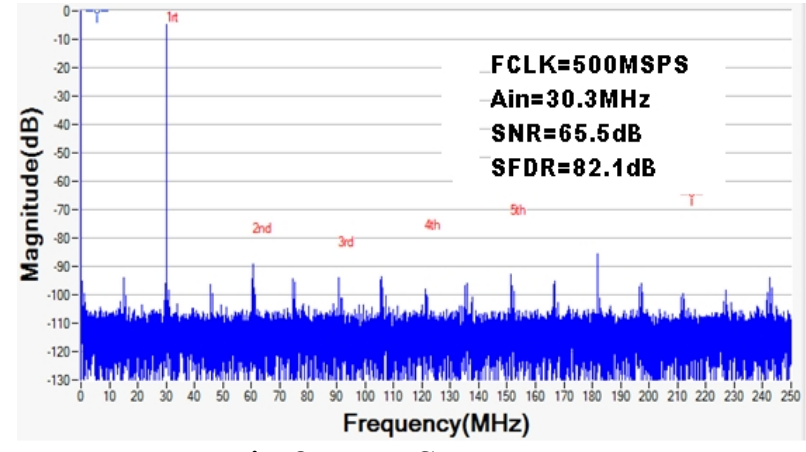

Fig.8 FFT Spectrum

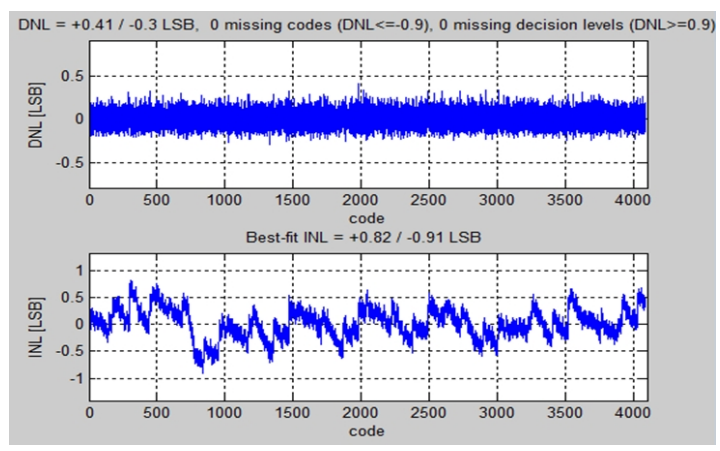

Fig.9 DNL and INL

\section{References}

[1] Ahmed M. A. Ali, Christopher Dillon, Robert Sneed, Andrew S. Morgan, Scott Bardsley, John Kornblum, Lu Wu; "A 14-bit $125 \mathrm{MS} / \mathrm{s} \mathrm{IF/RF} \mathrm{sampling} \mathrm{pipelined} \mathrm{ADC} \mathrm{with} 100 \mathrm{~dB}$ SFDR and 50 fs jitter”, IEEE Journal of Solid-State Circuits, vol. 41, pp. 1846 - 1855, August 2006.

[2] Alfio Zanchi and Dong-Young Chang, IEEE Trans. Circuits Syst, p.2166 (2008)

[3] C. Y. Chen et al., "A 12-bit 3GS/s pipeline ADC with $0.4 \mathrm{~mm}$ and $500 \mathrm{~mW}$ in $40 \mathrm{~nm}$ digital CMOS" Journal of Solid-State Circuits,vol. 47,no.4,pp.1013-1021,Apr.2012.

[4] B.Setterberg et al., "A 14b 2.5GS/s 8-way-interleaved pipelined ADC with background calibration and digital dynamic linearity correction," in IEEE ISSCC Dig.Tech.papers,2013,pp.466-468. 